\title{
Efficient Binary Linear Programming Formulations for Boolean Functions
}

\author{
Frank Gurski ${ }^{1, *}$ \\ ${ }^{1}$ Institute of Computer Science, Heinrich-Heine University of Düsseldorf, Germany
}

Received: 25 May 2014; Accepted: 3 September 2014

Editor: Houduo Qi

\begin{abstract}
A very useful tool when designing linear programs for optimization problems is the formulation of logical operations by linear programming constraints. We give efficient linear programming formulation of important $n$-ary boolean functions $f\left(x_{1}, \ldots, x_{n}\right)=x_{n+1}$ such as conjunction, disjunction, equivalence, and implication using $n+1$ boolean variables $x_{1}, \ldots, x_{n+1}$. For the case that the value $f\left(x_{1}, \ldots, x_{n}\right)$ is not needed for further computations, we even give a more compact formulation. Our formulations show that every binary boolean function $f\left(x_{1}, x_{2}\right)=x_{3}$ can be realized by the only three boolean variables $x_{1}, x_{2}, x_{3}$ and at most four linear programming constraints.
\end{abstract}

Keywords Binary linear programming; Boolean functions

AMS 2010 subject classifications 90C05,06E30

DOI: $10.19139 /$ soic.v2i4.83

\section{Introduction}

Linear programming is a powerful tool, studied for over 50 years, that can be used to define a lot of very important optimization problems [3, 6]. In a linear programming problem $(L P)$ we are given a linear function $f: \mathbb{R}^{n} \mapsto$ $\mathbb{R}, f\left(x_{1}, \ldots, x_{n}\right)=c_{1} x_{1}+\ldots+c_{n} x_{n}=\sum_{i=1}^{n} c_{i} x_{i}$. Function $f$ is denoted as objective function of the LP. Additionally, a set of constraints is given. In general a constraint either is an equality or an inequality which contains a

\footnotetext{
*Correspondence to: Heinrich-Heine University of Düsseldorf, Institute of Computer Science, Algorithmics for Hard Problems Group, D-40225 Düsseldorf, Germany. E-mail: frank.gurski@hhu.de
}

ISSN 2310-5070 (online) ISSN 2311-004X (print)

Copyright (C) 2014 International Academic Press 
linear combination of the variables of $f$, i.e. the $i$-th constraint is of the form $a_{i, 1} x_{1}+\ldots+a_{i, n} x_{n} \geq b_{i}$. Some of these constraints may be very simple, since they require that some of the variables are not negative, e.g., $x_{j} \geq 0$. Constraints of the first type are denoted as functional constraints and the latter ones are denoted as nonnegativity constraints.

A proposal $x_{1}^{\prime}, \ldots, x_{n}^{\prime}$ of values for the $n$ variables of $f$ is called a solution and the number $f\left(x_{1}^{\prime}, \ldots, x_{n}^{\prime}\right)$ its objective (function) value. A solution is denoted as feasible if it satisfies all constraints. A feasible solution $x$ is denoted as an optimal solution if the objective function value for $x$ is the smallest (minimization problem) or the largest value (maximization problem) over the objective function values for all feasible solutions. The linear programming problem is, given an objective function and a finite set of constraints, to find an optimal solution. Using matrices, a linear program can be expressed as a task of minimizing $c^{T} x$ subject to the constraints $A x \geq b$ and $x \geq 0$. This allows us to define the size of a linear program by $\operatorname{size}(A)+\operatorname{size}(b)+\operatorname{size}(c)$. The size of an integer (by multiplying the rational coefficients adequate we can assume that we have given integer coefficients) is the size of its binary representation. The size of a vector or of a matrix is the sum of the sizes of its elements.

In integer linear programming problems $(I P)$, the variables are all required to be integers and in binary linear programming problems $(B I P)$, each variable can only take the two values, zero or one. While general linear programming can be solved in polynomial time by interior point methods or the ellipsoid method [3], solving integer programming and even binary integer programming is NP-hard [5].

The task to find equivalent linear programming formulations for optimization problems is often challenging, see e.g. [7], [8], and [2]. In this connection it is often useful to have formulation of logical operations by linear programming constraints. Examples are linear programming formulations for graph parameters in [7], an ILP approach for register allocation in [1], and the allocation of logical constraints in LP-solvers as e.g. CPLEX.

In this paper we give efficient binary linear programming formulations (BIP formulations) for several important boolean functions such as $n$-ary conjunction, $n$-ary disjunction, equivalence, and implication. Our results also imply that every binary boolean function $f\left(x_{1}, x_{2}\right)=x_{3}$ can be realized by the three variables $x_{1}, x_{2}, x_{3}$ and at most four conditions, which was unknown until now.

\section{Realizing logic operations in linear programming}

We extend the definition of the binary boolean functions and and nor given in [7] and of the functions and, or, and not given in [2] and [4] to all possible 16 binary boolean functions (cf. Table I) and their generalizations to $n$-ary boolean functions. Let $x_{i}$ be boolean variables. 
Table I. All 16 binary boolean functions $f\left(x_{1}, x_{2}\right)$.

\begin{tabular}{|c|c|c|c|c|c|c|c|c|c|c|c|c|c|c|c|c|c|}
\hline$x_{1}$ & $x_{2}$ & 0 & $\begin{array}{l}\mathbb{E} \\
\dot{\mathbb{E}} \\
\dot{8}\end{array}$ & 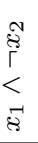 & $\vec{\xi}$ & 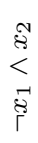 & ્ָळ & $\begin{array}{l}\tilde{C} \\
\oplus \\
\vec{\varnothing}\end{array}$ & $\begin{array}{l}\tilde{8} \\
> \\
\overrightarrow{8}\end{array}$ & $\begin{array}{l}\text { त्ष } \\
> \\
\overrightarrow{8} \\
\Gamma\end{array}$ & $\begin{array}{l}\mathbb{E} \\
\mathbb{1} \\
\overrightarrow{8}\end{array}$ & $\begin{array}{c}\tilde{C} \\
1 \\
-1\end{array}$ & $\begin{array}{l}\overrightarrow{8} \\
\Uparrow \\
\mathbb{E}\end{array}$ & $\begin{array}{l}\overrightarrow{8} \\
1 \\
-1\end{array}$ & $\begin{array}{l}\mathbb{8} \\
\Uparrow \\
\mathbb{8} \\
\overrightarrow{8}\end{array}$ & $\begin{array}{l}\text { तु } \\
< \\
\overrightarrow{\dot{E}} \\
\vec{\Gamma}\end{array}$ & -1 \\
\hline 0 & 0 & 0 & 0 & 0 & 0 & 0 & 0 & 0 & 0 & 1 & 1 & 1 & 1 & 1 & 1 & 1 & 1 \\
\hline 0 & 1 & 0 & 0 & 0 & 0 & 1 & 1 & 1 & 1 & 0 & 0 & 0 & 0 & 1 & 1 & 1 & 1 \\
\hline 1 & 0 & 0 & 0 & 1 & 1 & 0 & 0 & 1 & 1 & 0 & 0 & 1 & 1 & 0 & 0 & 1 & 1 \\
\hline 1 & 1 & 0 & 1 & 0 & 1 & 0 & 1 & 0 & 1 & 0 & 1 & 0 & 1 & 0 & 1 & 0 & 1 \\
\hline
\end{tabular}

\subsection{Negation}

A negation $x_{2}=\neg x_{1}$ can be realized by the following constraints.

$$
\begin{aligned}
x_{2} & =1-x_{1} \\
x_{1}, x_{2} & \in\{0,1\}
\end{aligned}
$$

Note that for boolean variables $x_{i}$, we can always substitute $x_{i}$ by $1-x_{i}$ within our conditions to realize the negation of $x_{i}$ without using the given additional constraint.

\subsection{Conjunction}

An $n$-ary conjunction (and) $x_{n+1}=\bigwedge_{i=1}^{n} x_{i}=x_{1} \wedge x_{2} \wedge \ldots \wedge x_{n}$ can be realized by $n+1$ constraints as follows.

$$
\begin{aligned}
x_{n+1} & \leq x_{1} \\
x_{n+1} & \leq x_{2} \\
\vdots & \vdots \vdots \\
x_{n+1} & \leq x_{n} \\
x_{1}+x_{2}+\ldots+x_{n} & \leq x_{n+1}+n-1 \\
x_{1}, x_{2}, \ldots, x_{n+1} & \in\{0,1\}
\end{aligned}
$$

The special case that we do not need the result of the $n$-ary conjunction for further computations in some variable and we only want to ensure that $\left(x_{1} \wedge x_{2} \wedge\right.$ $\left.\ldots \wedge x_{n}\right)$ is satisfied, can be realized by the single constraint $\sum_{i=1}^{n} x_{i} \geq n$.

Although our first two given formulations imply that every binary boolean function can be defined by linear programming constraints, we suggest the following conditions in order to get more efficient formulations and to prove Theorem 1. 


\subsection{Disjunction}

An $n$-ary disjunction (or) $x_{n+1}=\bigvee_{i=1}^{n} x_{i}=x_{1} \vee x_{2} \vee \ldots \vee x_{n}$ can be realized by $n+1$ constraints as follows.

$$
\begin{aligned}
x_{1} & \leq x_{n+1} \\
x_{2} & \leq x_{n+1} \\
\vdots & \vdots \vdots \\
x_{n} & \leq x_{n+1} \\
x_{1}+x_{2}+\ldots+x_{n} & \geq x_{n+1} \\
x_{1}, x_{2}, \ldots, x_{n+1} & \in\{0,1\}
\end{aligned}
$$

The special case that we do not need the result of the $n$-ary disjunction for further computations in some variable and we only want to ensure that $\left(x_{1} \vee x_{2} \vee\right.$ $\left.\ldots \vee x_{n}\right)$ is satisfied, can be realized by the single constraint $\sum_{i=1}^{n} x_{i} \geq 1$.

\subsection{Nand}

An $n$-ary nand $x_{n+1}=\neg\left(x_{1} \wedge x_{2} \wedge \ldots \wedge x_{n}\right)$ can be realized by $n+1$ constraints by substituting $x_{n+1}$ by $1-x_{n+1}$ in our solution for a $n$-ary conjunction.

The special case that we do not need the result of the $n$-ary nand for further computations in some variable and we only want to ensure that $\neg\left(x_{1} \wedge x_{2} \wedge \ldots \wedge\right.$ $\left.x_{n}\right)$ is satisfied, can be realized by the single constraint $\sum_{i=1}^{n} x_{i} \leq n-1$.

\subsection{Nor}

An $n$-ary nor $x_{n+1}=\neg\left(x_{1} \vee x_{2} \vee \ldots \vee x_{n}\right)$ can be realized by $n+1$ constraints by substituting $x_{n+1}$ by $1-x_{n+1}$ in our solution for a $n$-ary disjunction.

The special case that we do not need the result of the $n$-ary nor for further computations in some variable and we only want to ensure that $\neg\left(x_{1} \vee x_{2} \vee \ldots \vee\right.$ $\left.x_{n}\right)$ is satisfied, can be realized by the single constraint $\sum_{i=1}^{n} x_{i} \leq 0$.

\subsection{Exclusive Disjunction}

An exclusive disjunction $x_{3}=x_{1} \oplus x_{2}$ can also be defined by $x_{3}=\left(x_{1} \wedge\right.$ $\left.\neg x_{2}\right) \vee\left(\neg x_{1} \wedge x_{2}\right)$ and also by $x_{3}=\neg\left(x_{1} \Leftrightarrow x_{2}\right)$ or $x_{3}=\left(x_{1}+x_{2}\right) \bmod 2$ and 
be realized by four constraints.

$$
\begin{aligned}
x_{1}+x_{2}+x_{3} & \leq 2 \\
-x_{1}-x_{2}+x_{3} & \leq 0 \\
x_{1}-x_{2}+x_{3} & \geq 0 \\
-x_{1}+x_{2}+x_{3} & \geq 0 \\
x_{1}, x_{2}, x_{3} & \in\{0,1\}
\end{aligned}
$$

The special case that we do not need the result of the binary exclusive disjunction for further computations in some variable and we only want to ensure that $\left(x_{1} \oplus x_{2}\right)$ is satisfied, can be realized by the constraint $x_{1}+x_{2}=1$.

\subsection{Implication}

An implication $x_{3}=\left(x_{1} \Rightarrow x_{2}\right)$ can also be defined by $x_{3}=\left(\neg x_{1} \vee x_{2}\right)$ and thus be defined by the shown conditions for a binary disjunction and substituting $x_{1}$ by $1-x_{1}$ to realize $\neg x_{1}$.

$$
\begin{aligned}
1-x_{1} & \leq x_{3} \\
x_{2} & \leq x_{3} \\
1-x_{1}+x_{2} & \geq x_{3} \\
x_{1}, x_{2}, x_{3} & \in\{0,1\}
\end{aligned}
$$

The special case that we do not need the result of the implication for further computations in some variable and we only want to ensure that $\left(x_{1} \Rightarrow x_{2}\right)$ is satisfied, can be realized by the single constraint $x_{1} \leq x_{2}$.

\subsection{Equivalence}

An equivalence $x_{3}=\left(x_{1} \Leftrightarrow x_{2}\right)$ can also be defined by $x_{3}=\left(x_{1} \wedge x_{2}\right) \vee\left(\neg x_{1} \wedge\right.$ $\left.\neg x_{2}\right)$ or $x_{3}=\neg\left(x_{1} \oplus x_{2}\right)$ and thus be realized by substituting $x_{3}$ by $1-x_{3}$ in our solution for a binary exclusive disjunction.

$$
\begin{aligned}
x_{1}+x_{2}-x_{3} & \leq 1 \\
x_{1}+x_{2}+x_{3} & \geq 1 \\
-x_{1}+x_{2}+x_{3} & \leq 1 \\
x_{1}-x_{2}+x_{3} & \leq 1 \\
x_{1}, x_{2}, x_{3} & \in\{0,1\}
\end{aligned}
$$

The special case that we do not need the result of the equivalence for further computations in some variable and we only want to ensure that $\left(x_{1} \Leftrightarrow x_{2}\right)$ is satisfied, can be realized by the constraint $x_{1}=x_{2}$. 


\subsection{Binary Boolean Functions}

Our given formulations for logical operations imply for $n=2$ that all possible 16 binary boolean functions $f:\{0,1\}^{2} \rightarrow\{0,1\}$ which are summarized in Table 1 can be expressed very efficiently. The formulation of binary exclusive disjunction, or equivalently, the equivalence by only four conditions seems to be the hardest task within this result.

\section{Theorem 1}

Every binary boolean function $f:\{0,1\} \times\{0,1\} \rightarrow\{0,1\}, f\left(x_{1}, x_{2}\right)=x_{3}$ can be realized by three variables $x_{1}, x_{2}, x_{3}$ and at most four conditions.

\section{Conclusions and Outlook}

We have given efficient linear programming formulation of several important $n$ ary boolean functions. Our results extend the definition of the binary boolean functions and and nor given in [7] and of the functions and, or, and not given in [2] and [4] and allow us to show that every binary boolean function $f\left(x_{1}, x_{2}\right)=x_{3}$ can be realized by the only three boolean variables $x_{1}, x_{2}, x_{3}$ and at most four linear programming constraints. We suppose that for $n=2$ our solutions are best possible with respect to their size. For $n>2$ the problem gets much more extensive.

\section{REFERENCES}

1. A.W. Appel and L. George. Optimal Spilling for CISC Machines with Few Registers. In Proceedings of the ACM SIGPLAN 2001 Conference on Programming Language Design and Implementation, PLDI '01, pages 243-253. ACM, 2001.

2. D.-S. Chen, R.G. Batson, and Y. Dang. Applied Integer Programming: Modeling and Solution. Wiley, New York, 2010.

3. V. Chvátal. Linear Programming. W. H. Freeman and Company, New York, 1983.

4. FICO ${ }^{\mathrm{TM}}$. MIP formulations and linearizations - Quick reference. Technical report, Fair Isaac Corporation, Warwickshire CV32 5YN, UK, 2009.

5. M.R. Garey and D.S. Johnson. Computers and Intractability: A Guide to the Theory of NPCompleteness. W.H. Freeman and Company, San Francisco, 1979.

6. M. Jünger, T.M. Liebling, D. Naddef, G.L. Nemhauser, W.R. Pulleyblank, G. Reinelt, G. Rinaldi, and L.A. Wolsey, editors. 50 Years of Integer Programming 1958-2008. SpringerVerlag, 2010.

7. J. Luttamaguzi, M. Pelsmajer, Z. Shen, and B. Yang. Integer programming methods for several optimization problems in graph theory. In Proceedings of the International Conference on Computers and Their Applications, CATA 2005, pages 50-55. ISCA, 2005.

8. R. Vanderbei. Linear Programming. Springer-Verlag, Berlin, 2008. 